\title{
Mie excitons: Understanding strong coupling in dielectric nanoparticles
}

\author{
Tserkezis, C.; Gonçalves, P. A.D.; Wolff, C.; Todisco, F.; Busch, K.; Mortensen, N. A.
}

Published in:

Physical Review B

Link to article, DOI:

10.1103/PhysRevB.98.155439

Publication date:

2018

Document Version

Publisher's PDF, also known as Version of record

Link back to DTU Orbit

Citation (APA):

Tserkezis, C., Gonçalves, P. A. D., Wolff, C., Todisco, F., Busch, K., \& Mortensen, N. A. (2018). Mie excitons: Understanding strong coupling in dielectric nanoparticles. Physical Review B, 98(15), [155439].

https://doi.org/10.1103/PhysRevB.98.155439

\section{General rights}

Copyright and moral rights for the publications made accessible in the public portal are retained by the authors and/or other copyright owners and it is a condition of accessing publications that users recognise and abide by the legal requirements associated with these rights.

- Users may download and print one copy of any publication from the public portal for the purpose of private study or research.

- You may not further distribute the material or use it for any profit-making activity or commercial gain

- You may freely distribute the URL identifying the publication in the public portal 


\title{
Mie excitons: Understanding strong coupling in dielectric nanoparticles
}

\author{
C. Tserkezis,,${ }^{1,}$ P. A. D. Gonçalves,,${ }^{1,2,3}$ C. Wolff, ${ }^{1}$ F. Todisco,,${ }^{1}$ K. Busch,,${ }^{4,5}$ and N. A. Mortensen ${ }^{1,2,6, \dagger}$ \\ ${ }^{1}$ Center for Nano Optics, University of Southern Denmark, Campusvej 55, DK-5230 Odense M, Denmark \\ ${ }^{2}$ Center for Nanostructured Graphene, Technical University of Denmark, DK-2800 Kongens Lyngby, Denmark \\ ${ }^{3}$ Department of Photonics Engineering, Technical University of Denmark, DK-2800 Kongens Lyngby, Denmark \\ ${ }^{4}$ Max-Born-Institut, Max-Born-Straße 2A, D-12489 Berlin, Germany \\ ${ }^{5}$ Humboldt-Universität zu Berlin, Institut für Physik, AG Theoretische Optik \& Photonik, Newtonstraße 15, D-12489 Berlin, Germany \\ ${ }^{6}$ Danish Institute for Advanced Study, University of Southern Denmark, Campusvej 55, DK-5230 Odense M, Denmark
}

(Received 15 July 2018; revised manuscript received 16 September 2018; published 26 October 2018)

\begin{abstract}
We theoretically analyze the hybrid Mie-exciton optical modes arising from the strong coupling of excitons in organic dyes or transition-metal dichalcogenides with the Mie resonances of high-index dielectric nanoparticles. Detailed analytic calculations show that silicon core-exciton shell nanoparticles are characterized by a richness of optical modes, which can be tuned through nanoparticle dimensions to produce large anticrossings in the visible or near infrared, comparable to those obtained in plexcitonics. The complex magnetic-excitonic nature of these modes is understood through spectral decomposition into Mie-coefficient contributions, complemented by electric and magnetic near-field profiles. In the frequency range of interest, absorptive losses in silicon are sufficiently low to allow observation of several periods of Rabi oscillations in strongly coupled emitter-particle architectures, as confirmed here by discontinuous Galerkin time-domain calculations for the electromagnetic field beat patterns. These results suggest that Mie resonances in high-index dielectrics are promising alternatives for plasmons in strong-coupling applications in nanophotonics, with the coupling of magnetic and electric modes potentially providing additional freedom for external control, whilst offering new possibilities for tailoring artificial optical magnetism.
\end{abstract}

DOI: 10.1103/PhysRevB.98.155439

\section{INTRODUCTION}

The interaction of optical modes in a structured electromagnetic (EM) environment with the photons emitted by atoms, molecules, organic dyes, quantum dots, or nanomaterial defects has long been in the forefront of interest in photonics, as it is characterized by novel fundamental physics and exciting applications [1-7]. Plasmons, in particular, are frequently combined with classical and quantum emitters, and are acknowledged as excellent templates for sensing [8,9], fluorescence [10,11] and Raman enhancement [12], and optical communications $[13,14]$. Recently, strong coupling of emitters with surface plasmon polaritons in metal films or localized surface plasmons in nanoparticles (NPs) has turned into a rapidly growing field, due to its potential for applications in quantum optics [15-18]. In the so-called plexcitonic architectures, plasmons tightly confine light to subwavelength volumes, thus largely overcoming the diffraction limit [19], dramatically enhancing the coupling strength and enabling light-matter interactions to enter the strong coupling regime, which is characterized by Rabi oscillations in the emitter occupation and hybrid optical states of mixed light-matter nature [20-25]. Nevertheless, full implementation of plasmonic designs in applications is still hindered by their high ohmic losses [26,27], and different schemes are explored.

\footnotetext{
*ct@mci.sdu.dk

†asger@mailaps.org
}

Among the various materials encountered in nanophotonics, high-refractive-index dielectrics hold a prominent position, as they combine low loss with-unavailable in metalsmagnetic Mie modes and compatibility with existing nanoelectronic platforms [28-30]. Silicon NPs, in particular, have been successfully exploited as building blocks for nanoantennas, sensing environments and optical metamaterials [31,32]. These advances have shown that on many occasions dielectric NPs do not fall short in comparison to their plasmonic counterparts [33,34]. It is therefore natural to consider them as prospective templates for strong coupling designs. Recently, it was shown that resonance coupling in heteroaggregates of dyes combined with high- [35] or moderate-index dielectric NPs [36] is indeed possible, while coupling with the anapole modes of silicon nanodiscs has also been reported [37]. Nevertheless, a thorough investigation and theoretical understanding of the nature of the resulting hybrid polaritons is still missing.

Here we present a theoretical study of the hybrid Mieexciton modes that arise from the interaction of magnetic dipolar modes in silicon nanospheres and nanoshells with the excitons sustained by $J$ aggregates of organic molecules or two-dimensional (2D) transition-metal dichalcogenides (TMDs) $[38,39]$. Through analytic solutions based on Mie theory [40], combined with discontinuous Galerkin timedomain (DGTD) dynamical studies [41,42], we show that mode splittings of the order of $150-200 \mathrm{meV}$ can be achieved by coupling emitters to dielectric NPs. Far- and near-field analysis shows that the resulting modes are characterized by a 
mixed, electric/magnetic dipolar nature, while the periods of the EM field temporal oscillations (analogous to Rabi oscillations in two-level systems) are in perfect agreement with the frequency splitting. Comparison with plasmonic architectures with similar spectral anticrossings shows that in the latter the beat patterns decay much faster. Mie excitons are therefore efficient alternatives to plexcitonics, thereby envisaging lowloss and externally controllable strong-coupling templates for applications in nanophotonics.

\section{RESULTS AND DISCUSSION}

In this work, we focus on the coupling of excitons with the Mie resonances sustained by isolated, noninteracting NPs. Single NPs significantly facilitate theoretical analysis, and are frequently encountered in experiments, not only in plasmonics [23,43] but also in the case of dielectrics [35,44]. The situation can become more complex in ensembles of interacting NPs, where a combination of effects including inhomogeneous broadening due to size variations and imperfections [45,46], multiple scattering and transport in random media [47-49], Anderson localization [50,51], and EM forceinduced clustering [52], is expected to play an important role.

The silicon-exciton NPs considered here comprise a homogeneous spherical core of radius $R_{1}$ and a concentric shell of thickness $D$, so that the total radius is $R=R_{1}+D$, as schematically shown in Fig. 1(a). Both arrangements of core and shell materials are allowed, while air is the host medium throughout the paper. Silicon permittivity $\left(\varepsilon_{\mathrm{Si}}\right)$ follows the experimental values of Ref. [53], so as to provide a dispersive and lossy description of its optical response (silicon loss is negligible in the infrared, but cannot be ignored in the visible). For the generic excitonic material, we use a (frequency, $\omega$, dependent) Lorentz dielectric function,

$$
\varepsilon_{\mathrm{exc}}(\omega)=\varepsilon_{\infty}-\frac{f \omega_{\mathrm{exc}}^{2}}{\omega^{2}-\omega_{\mathrm{exc}}^{2}+\mathrm{i} \omega \gamma_{\mathrm{exc}}},
$$

where $\omega_{\mathrm{exc}}$ is the excitonic transition frequency, $\gamma_{\mathrm{exc}}$ the corresponding damping rate, $f$ the oscillator strength, and $\varepsilon_{\infty}$ the background permittivity. Choosing $\hbar \omega_{\mathrm{exc}}=1.76 \mathrm{eV}$, $\hbar \gamma_{\mathrm{exc}}=0.05 \mathrm{eV}, f=0.4$, and $\varepsilon_{\infty}=3$ accounts fairly well for the resonance in the dielectric function of squaraine, while disregarding broadening at higher energies to facilitate theoretical analysis. A dielectric function with such a large oscillator strength has proved sufficient to lead to the ultrastrong coupling regime in the context of plexcitonics [54]. In general, smaller oscillator strengths are expected to lead to narrower mode splittings [55,56].

We first consider an excitonic core-silicon shell NP with $R_{1}=70 \mathrm{~nm}$ and $R=100 \mathrm{~nm}$. Such a design, though challenging from an experimental point of view, is beneficial to theoretical understanding, as it reduces mode mixing. Indeed, as can be seen by decomposing the extinction cross section $\left(\sigma_{\text {ext }}\right.$, normalised to the geometrical cross section) in Fig. 1(a), when the excitonic resonance of the core is disregarded [ $f=0$ in Eq. (1)], the silicon shell is characterised by well-defined magnetic Mie resonances of increasing multipolar order (blue solid and dashed lines for the dipolar and quadrupolar modes, respectively), over the tail of a wide but weak electric dipolar background (red solid line). This is in

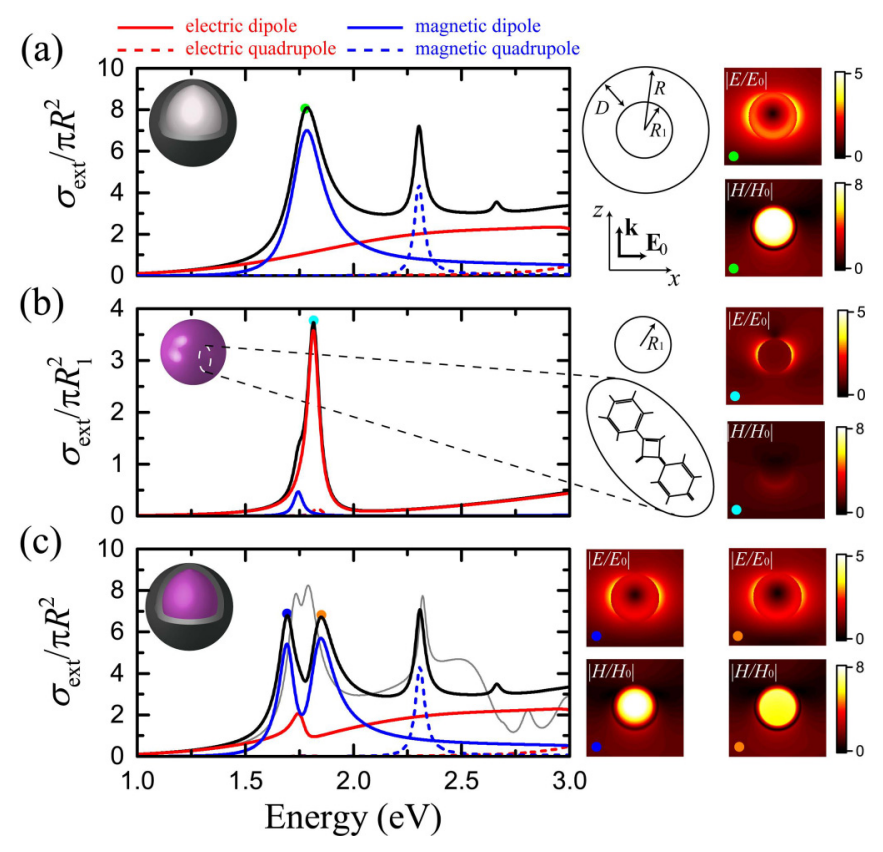

FIG. 1. (a) Normalized extinction cross section ( $\sigma_{\text {ext }}$, black line) of the uncoupled [ $f=0$ in Eq. (1) for the core, $\varepsilon=\varepsilon_{\mathrm{Si}}$ for the shell] core-shell NP of the inset $\left(R_{1}=70 \mathrm{~nm}\right.$ and $\left.R=100 \mathrm{~nm}\right)$, and the contributions from the electric (red) and magnetic (blue) dipolar (solid lines) and quadrupolar (dashed lines) Mie coefficients. Electric and magnetic field amplitude profiles (in the $x z$ plane, normalised to the incident fields), $\left|E / E_{0}\right|$ and $\left|H / H_{0}\right|$, respectively, at the frequency of the magnetic dipolar resonance (green dot in the spectra) are shown on the right-hand side, for an incident plane wave propagating along the $z$ and polarized along the $x$ axis. (b) Same as in (a), for an excitonic sphere $\left(R_{1}=70 \mathrm{~nm}\right)$ described by the dielectric function of Eq. (1). The near-field profiles are plotted at the frequency indicated by the light-blue dot. (c) Analysis of $\sigma_{\text {ext }}$, and corresponding field profiles at the frequencies indicated by the blue and orange dots in the spectra, for the coupled $[f=0.4$ in Eq. (1)] exciton core-silicon shell NP $\left(R_{1}=70 \mathrm{~nm}\right.$ and $\left.R=100 \mathrm{~nm}\right)$. The corresponding extinction spectrum for $R_{1}=40 \mathrm{~nm}$ and $R=91 \mathrm{~nm}$ is shown by a thin grey line.

contrast to the case of homogeneous silicon spheres, where the electric dipole mode is pronounced and strongly overlaps with the low-energy magnetic dipole one [28] [see also Fig. 2(a)].

The magnetic dipole nature of the first mode, at about $1.79 \mathrm{eV}$, is further confirmed by the electric and magnetic field amplitude profiles $\left(\left|E / E_{0}\right|\right.$ and $\left|H / H_{0}\right|$, normalized to the corresponding incident field) on the right-hand side.

These near-field profiles also indicate that the uncoupled dielectric NPs are characterized by rather extended mode volumes. Since the coupling strength is proportional to $\sqrt{N / V}$, where $N$ is the number of emitters and $V$ the mode volume of the (here open) cavity [15], one anticipates that roomtemperature single-molecule strong coupling should be rather challenging to achieve: only with the extreme volume minimization of plasmonics has this been reported so far [43]. Nevertheless, high concentrations of emitters, e.g., in the form of molecular $J$-aggregates, are still suitable candidates for such applications. 


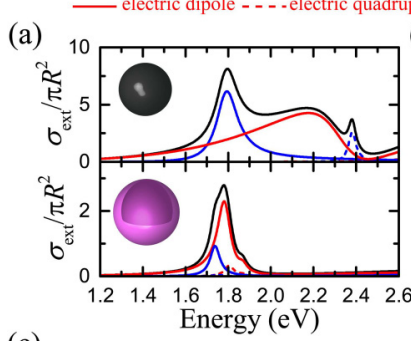

(c)
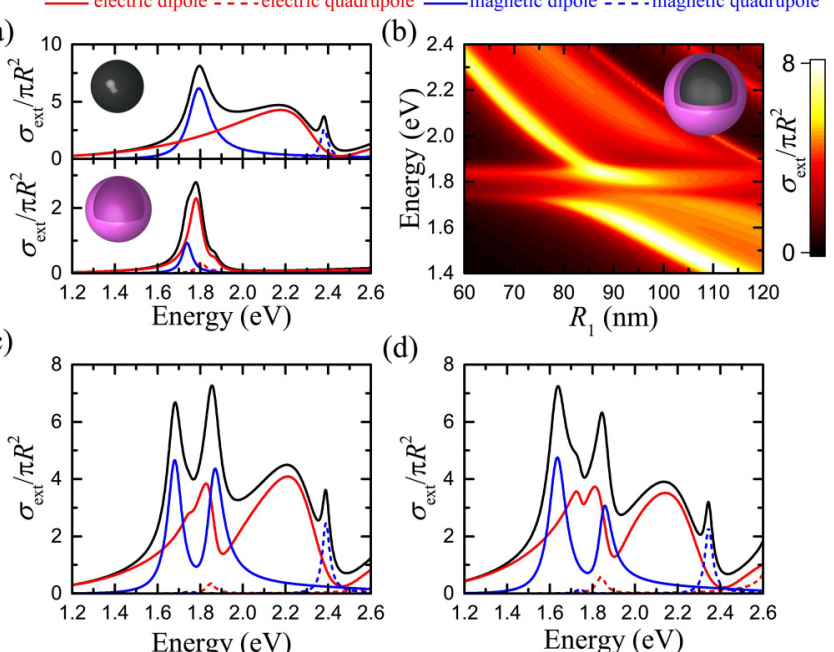

(d)

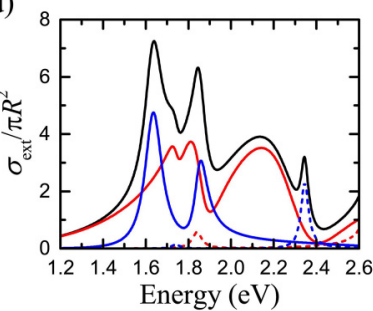

FIG. 2. (a) Upper panel: normalized extinction cross section of a silicon sphere $\left(R_{1}=85 \mathrm{~nm}\right)$ in an environment with permittivity $\varepsilon=$ 3 (black line). Lower panel: normalized extinction cross section of an excitonic shell of $R_{1}=85 \mathrm{~nm}$ and $D=20 \mathrm{~nm}$ (assuming a vacuum core), described by the dielectric function of Eq. (1) (black line). In both panels we also show the contributions from the electric (red) and magnetic (blue) dipolar (solid lines) and quadrupolar (dashed lines) Mie coefficients. (b) Extinction color map as a function of the core radius $R_{1}$ of the silicon core-exciton shell nanosphere shown in the inset $(D=20 \mathrm{~nm})$. Anticrossing is obtained for $R_{1} \simeq 85 \mathrm{~nm}$. (c) Mie-coefficient analysis of the anticrossing spectra $\left(R_{1}=85 \mathrm{~nm}\right.$, $R=105 \mathrm{~nm})$. (d) Same as (c), for a thicker excitonic shell $\left(R_{1}=\right.$ $87 \mathrm{~nm}, D=30 \mathrm{~nm})$.

The almost negligible contribution of the electric dipole mode allows to verify whether the magnetic dipole mode of the silicon shell can significantly interact with the electric dipole of the excitonic core, once tuned to coincide in frequency. The extinction spectrum of the bare excitonic core $\left(R_{1}=70 \mathrm{~nm}\right)$, described by the dielectric function of Eq. (1), is shown in Fig. 1(b), while the near-field profiles on resonance appear on the right-hand side. For such a large core size, a second, magnetic dipolar mode is also excited, and appears as a shoulder in the extinction spectrum. A smaller radius would ensure excitation of a single electric dipolar mode, but our calculations showed that in that case $\left(R_{1} \lesssim\right.$ $40 \mathrm{~nm}$ ) the core dipole moment is not strong enough to support the desired coupling strength, as discussed in the previous paragraph. This will become clearer below, through direct comparison of the black (thick excitonic core, larger emitter number) and grey (thinner core, smaller emitter number) spectra of the coupled NP in Fig. 1(c).

When the two components are merged in a core-shell geometry, as depicted in Fig. 1(c), their modes couple like harmonic oscillators [15], leading to a double-peak spectrum where the hybrid Mie-exciton modes are separated by a split of $156 \mathrm{meV}$, comparable to the linewidth of the silicon shell resonance in Fig. 1(a) (145 meV). This split just satisfies the common criterion for strong coupling, i.e., that it must be larger than half the sum of the uncoupled linewidths squared. A detailed discussion about the different criteria associated to strong coupling, both in nanophotonics and in

other disciplines, can be found in Ref. [15]. The resulting hybrid modes combine the electric and magnetic characters of the uncoupled components, but maintain a dominantly magnetic dipolar nature due to the relative differences in the dipole strengths of the original modes. This is verified both by the Mie-coefficient analysis and the near-field profiles on the right-hand side. The field profiles are not identical, because of the asymmetric electric dipole background provided by the silicon shell [Fig. 1(a)], but the magnetic field enhancement inside the otherwise nonmagnetic NP core is evident. A more symmetric extinction spectrum with equal linewidths for the two hybrid modes can be achieved by fine, subnanometer tuning of the core and shell radii.

In what follows we invert the material arrangement and consider Si nanospheres, whose spectra resemble those shown in the upper panel of Fig. 2(a) [for $R_{1}=85 \mathrm{~nm}$, in an environment described by Eq. (1) with $f=0$ ] [28], covered with homogeneous excitonic shells. Thick shells sustain not only the exciton resonance at fixed frequency $\omega_{\text {exc }}$, but also geometrical modes corresponding to a curved NP with a negative dielectric function, that become stronger as the shell thickness increases [55,57], similarly to what one expects for plasmonic NPs [in the spectra of the bottom panel of Fig. 2(a) $(D=20 \mathrm{~nm})$ such a mode has just appeared as a shoulder at $1.85 \mathrm{eV}]$.

Nevertheless, the optical response can still be precisely tuned by adjusting the silicon core. In Fig. 2(b), we increase $R_{1}$ (for constant $D=20 \mathrm{~nm}$ ) to shift the magnetic dipole mode of the core from the infrared (large NPs) all the way to the visible. When it matches the excitonic resonance of the shell (for $R_{1}=85 \mathrm{~nm}$, with a linewidth of $97 \mathrm{meV}$ ), an anticrossing of $178 \mathrm{meV}$ emerges [Fig. 2(c)], indicating strong coupling. Wider splits, exceeding $200 \mathrm{meV}$ can be achieved by increasing the dipole moment of the excitonic layer, e.g., by increasing $f$ in Eq. (1), or, for fixed permittivity, by increasing the shell thickness, as shown in Fig. 2(d) $\left(R_{1}=\right.$ $87 \mathrm{~nm}$ and $D=30 \mathrm{~nm}$ ). However, in the latter situation the geometrical shell modes are more pronounced, appearing as additional peaks or shoulders in the spectra, and the mode splitting is not well-defined [55]. Furthermore, in Fig. 2(d), two different couplings can be identified in the Mie coefficients, with different splits in the magnetic and electric dipole contribution. This complex interaction originates from the fact that the shell now sustains two modes, close in frequency but with comparable strengths and linewidths [bottom panel in Fig. 2(a)], which both interact with the silicon core. We also note that the middle peak (the shoulder at $1.7 \mathrm{eV}$ ) is mainly absorptive, as one can see in Figs. 3(a) and 3(b), where we decompose the corresponding extinction spectra of Figs. 2(c) and 2(d) into their scattering and absorption contributions, $\sigma_{\text {scat }}$ and $\sigma_{\text {abs }}$, respectively. In both cases the absorption spectra are characterized by about $10 \mathrm{meV}$ narrower splits compared to their scattering counterparts, in agreement with previous works, where the need for care when discussing strong coupling through scattering or absorption spectra has been stressed $[55,58,59]$.

A key element in strong coupling studies is the period and decay of Rabi oscillations in the occupation of two-level systems. For our excitonic layers, such an occupation is not strictly defined, but important information can be retrieved by 


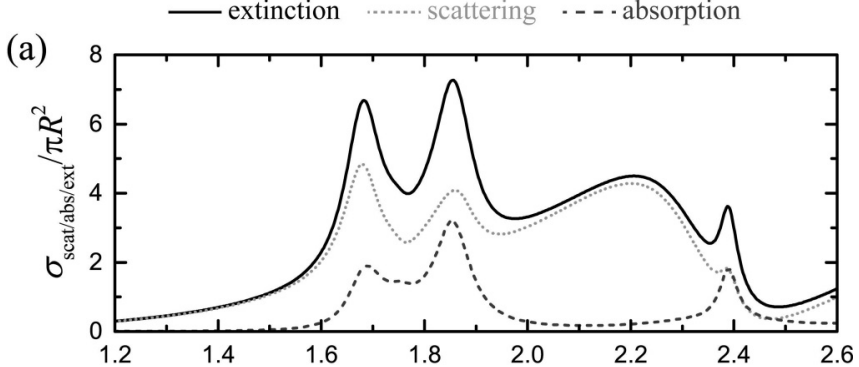

(b)

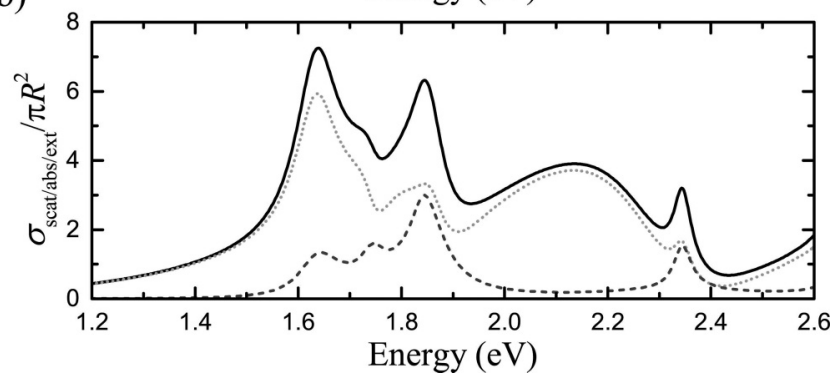

FIG. 3. (a) Analysis of the extinction spectra of Fig. 2(c) in terms of their scattering (light-grey dotted line) and absorption (grey dashed line) contributions. (b) Same as in (a), for the spectra of Fig. 2(d).

the time evolution of the EM field around the NP. The beat pattern for the $x$ component of the electric field $\left(E_{x}\right)$ at $25 \mathrm{~nm}$ from the NP surface, obtained with the DGTD method [60], is shown by a black line on the right-hand side of Fig. 4(a) for the silicon core-exciton shell NP of Fig. 2(c).

For the dynamic calculations, the NP and surrounding air and perfectly matched layer termination were discretized into 19500 tetrahedral elements with third-order Lagrange polynomials as basis functions (element size $15 \mathrm{~nm}$ inside the $\mathrm{NP}, 50 \mathrm{~nm}$ in air). The setup was excited by a pulse with ( $x$ polarized) plane-wave transversal profile and a narrow-band Gaussian envelope [carrier wavelength $700 \mathrm{~nm}$, full width at half-maximum (FWHM) $16 \mathrm{fs}$. The experimental permittivity of silicon [53] was fitted with a Lorentzian dielectric function similar to that of Eq. (1) (see Ref. [61] for the details), with excellent agreement (relative error less than 5\%) up to $3.3 \mathrm{eV}$. The obtained oscillations are accurately described by an envelope function (red line) of an exponentially decaying cosine (note that the first 25-30 fs correspond to NP excitation by the incident pulse, and are not considered as part of the NP response described by the envelope function), whose frequency exactly matches the mode splitting ( $\hbar \omega=0.179 \mathrm{eV}$ ) of the left-hand spectra. Similar beat patterns were obtained for the magnetic field inside the NP [see lower diagram on the right-hand side of Fig. 4(a), for the $y$ component of the magnetic field, $H_{y}$, at the NP center]. Interestingly, while the magnetic field profiles of the hybrid modes are similar, their electric field distributions are very different [middle panel in Fig. 4(a)], most notably regarding the field enhancement inside the shell for the high-energy branch. In view of our discussion of Fig. 1, the differences can be attributed to the asymmetric background originating from additional, higherorder modes in the frequency region of the split.
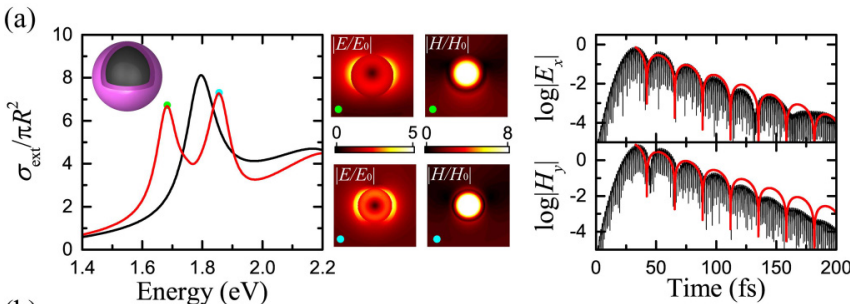

(b)
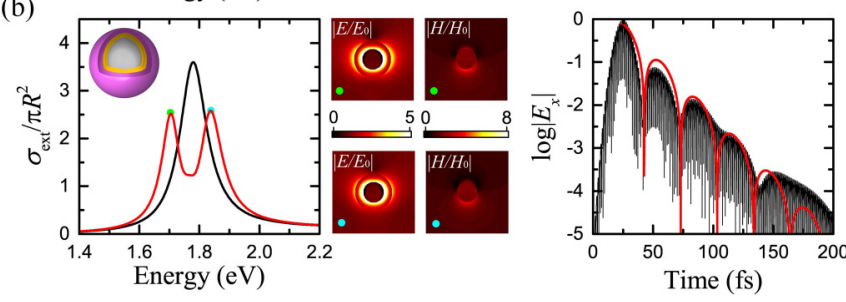

FIG. 4. (a) (Left) extinction spectra for the uncoupled [black line, $f=0$ in Eq. (1)] and coupled (red line) silicon core-exciton shell $\mathrm{NP}$ of the inset [NP dimensions are as in Fig. 2(b)]. (Middle) electric and magnetic field profiles in the $x z$ plane, for an $x$-polarized plane wave at the frequencies of the two coupled Mie excitons (denoted by the green and light-blue dots in the spectra of the left-hand panel). (Right, top) Time dependence of the logarithm of the $x$ component of the total (incident + scattered) electric field (black line), at $25 \mathrm{~nm}$ from the NP surface along the $x$ axis, when the NP is irradiated by a Gaussian envelope with 16 fs FWHM (carrier wavelength $700 \mathrm{~nm}$ ) and plane-wave transversal profile. The red line represents the corresponding envelope function. The bottom diagram shows the corresponding beat pattern for the $y$ component of the magnetic field, $H_{y}$, calculated at the NP center. (b) Same as in (a), for a silicagold-dye trilayered NP [silica core radius $19.5 \mathrm{~nm}, R_{1}=25 \mathrm{~nm}$, $D=20 \mathrm{~nm}$ (only the electric field beat pattern is relevant in this case)].

To explore similarities and advantages of silicon NPs over their plasmonic counterparts, we compare in Fig. 4 the extinction spectra, electric field profiles, and beat patterns, with those of an exciton-covered gold NP. As before, the dynamic response was simulated using DGTD with a similar mesh with 27500 elements (element size in the metal $6 \mathrm{~nm}$ ) and modeling the dielectric properties of gold [62] through a Drude-Lorentz fit with maximal relative error of $6 \%$ over the spectral range 0.6-3.1 eV (see Ref. [63] for numeric values; the same model was used in the frequency-domain calculations for consistency):

$$
\varepsilon_{\mathrm{Au}}=\varepsilon_{\infty}^{\mathrm{Au}}-\frac{\omega_{\mathrm{p}}^{2}}{\omega^{2}+\mathrm{i} \omega \gamma_{\mathrm{p}}}-\sum_{i=1}^{2} \frac{f_{i} \omega_{\mathrm{L} i}^{2}}{\omega^{2}-\omega_{\mathrm{L} i}^{2}+\mathrm{i} \omega \gamma_{\mathrm{L} i}}
$$

For a meaningful comparison, one should have the same geometrical parameters, extinction spectra (in terms of resonance position and linewidth), scattering and absorption contributions, and mode anticrossing. Since this is practically impossible, we focus here on a NP with similar linewidth and resonance position of its lower-energy mode before couplingin plasmonics, this is of course an electric dipolar mode. To tune the plasmon mode at $1.76 \mathrm{eV}$, we consider silicagold nanoshells $\left(\varepsilon_{\text {silica }}=2.13\right)$ instead of homogeneous gold spheres and introduce plasmon hybridization as a mode shifting mechanism [64,65]. For a silica core of radius $19.5 \mathrm{~nm}$, 
total nanoshell radius ( $R_{1}$ in the previous context) of $25 \mathrm{~nm}$, and $D=20 \mathrm{~nm}$, the extinction spectrum of Fig. 4(b) is calculated. When the exciton resonance is introduced $[f \neq 0$ in Eq. (1)], a 136-meV split is readily obtained. This frequency provides an envelope function that again excellently describes the temporal profile of the electric field (right-hand panel). More importantly, fewer [as compared to Fig. 4(a)] periods of oscillations are observable before complete dissipation (a reduction of the field by three orders of magnitude is produced already after $100 \mathrm{fs}$ ), which implies that dielectric NPs are more suitable candidates for dynamical studies of strong coupling. Another striking difference can be seen in the field profiles [middle panel in Fig. 4(b)] in the plasmonic case, where a single electric dipole mode interacts with the exciton of the dye and the resulting hybrid modes are practically identical, in contrast to the rather asymmetric field distributions shown in Fig. 4(a).

Finally, as an alternative, more robust and easily achievable with current nanofabrication techniques design, we replace the shell of organic molecules with a monolayer of TMD, specifically $\mathrm{WS}_{2}$, as was recently done in Refs. [44,66]. Atomically thin semiconductors based on TMDs-with chemical formula $M X_{2}$, where $M=\{\mathrm{W}, \mathrm{Mo}\}$ and $X=\{\mathrm{S}, \mathrm{Se}, \mathrm{Te}\}-$ exhibit strong light-matter interactions [67,68], at the heart of which lie excitons with large binding energies due to the weaker screening resulting from the reduced dimensionality [69]. The optical response of TMDs in the visible and near-infrared is dominated by excitonic resonances, even at room temperature [70], which, combined with their tunability (provided by layer number, strain, gating, etc.), makes them attractive platforms for strong coupling under ambient conditions [71]. Fabrication of gold nanospheres coated with few-to-a-single $\mathrm{MoS}_{2}$ layers has been experimentally demonstrated in a number of recent studies [72-75]. Furthermore, a monolayer of $\mathrm{WS}_{2}$ deposited on a metal film was recently shown to lead to mode splittings of the order of $70-80 \mathrm{meV}$, while even higher values can be achieved by engineering the dielectric environment [38]. For our purposes, such an excitonic material provides a "cleaner" system, with just a single excitonic resonance in the spectral window of interest.

For the dielectric function of $\mathrm{WS}_{2}, \varepsilon_{2 \mathrm{D}}$, we use the experimental data of Ref. [70], fitted with a multioscillator Lorentzian, as described in Ref. [38]. Within Mie theory, the 2D material is introduced as a surface current and corresponding conductivity to the boundary conditions (see Appendix for details). In Fig. 5(a), we show extinction spectra for $\mathrm{WS}_{2}$ covered silicon NPs as the core radius is modified. Clearly, an avoided crossing emerges around the $\mathrm{WS}_{2}$ excitonic resonance (i.e., at $2.01 \mathrm{eV}$ for the so-called $A$ exciton) [76]. The corresponding uncoupled and coupled spectra at the crossing point (for $R_{1}=75 \mathrm{~nm}$ ) are shown in Fig. 5(b) (black and red line for the bare $\mathrm{WS}_{2}$-covered silicon NP, respectively), exhibiting a relatively narrow split, of about about $50 \mathrm{meV}$. Size and shape engineering should enable stronger mode hybridization, while 2D TMDs can even be used as substrates or covering layers of photonic nanostructures.

Another possible route to achieve larger coupling strengths is by using few-layer TMDs in order to increase the effective interaction volume. Indeed, and despite the fact that the TMD band gap becomes indirect as the layer number increases, this
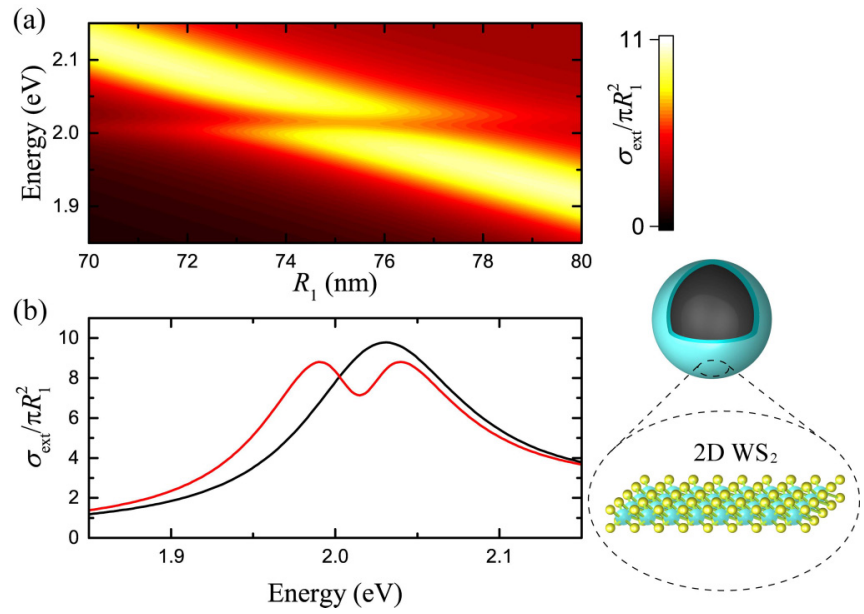

FIG. 5. (a) Extinction contour as a function of the radius $R_{1}$ of the silicon core, for the silicon-WS $\mathrm{W}_{2}$-monolayer NP shown schematically on the right-hand side. (b) Extinction spectra of a bare silicon $\mathrm{NP}$ (black line) and a $\mathrm{WS}_{2}$-coated silicon NP (red line) near the crossing point $\left(R_{1}=75 \mathrm{~nm}\right)$.

approach was proven to be successful in a recent experimental study involving plasmonic NPs on a planar surface covered with $\mathrm{WSe}_{2}$ [77]. Nevertheless, this procedure has to be carried out judiciously since upon the addition of more layers the band structure of TMDs typically undergoes considerable changes [69].

\section{CONCLUSIONS}

We explored the coupling of excitons in organic molecules and 2D TMDs with the magnetic Mie modes of silicon NPs. Such complex nanostructures are characterized by rich optical spectra, dominated by hybrid Mie-exciton modes, with splits comparable to those seen in plexcitonics. Nevertheless, attention is required when analyzing far-field spectra, as additional modes can mix with the Mie excitons and manifest themselves in scattering, in absorption, or in both. Near-field profiles verify that these modes combine the electric and magnetic field enhancements of their constituents, and could potentially facilitate external control with an applied bias or magnetic field, while they also offer an alternative route to controlling artificial optical magnetism [78,79], through the splitting of a single magnetic resonance. Direct comparison between the time evolution of the fields around silicon- or gold-exciton core-shell NPs shows that dielectrics are more efficient substitutes for metals when studying strong coupling dynamics. Finally, we suggest alternative architectures based on TMDcovered high-index NPs, which provide more flexibility and tunability in practical realizations.

\section{ACKNOWLEDGMENTS}

We thank V. A. Zenin for discussions. N. A. Mortensen is a VILLUM Investigator supported by VILLUM FONDEN (Grant No. 16498). The Center for Nano Optics is financially supported by the University of Southern Denmark (SDU 2020 funding). The Center for Nanostructured Graphene is sponsored by the Danish National Research 
Foundation (Project No. DNRF103). F. Todisco and C. Wolff acknowledge funding from MULTIPLY fellowships under the Marie Skłodowska-Curie COFUND Action (grant agreement No. 713694). K. Busch acknowledges support by the Deutsche Forschungsgemeinschaft within the frame of project B10 of SFB 951 (HIOS).

\section{APPENDIX: MIE THEORY FOR A SPHERE COATED WITH A 2D MATERIAL}

Most of the calculations in this paper were performed with standard Mie theory, the full derivation of which can be found in several textbooks [40]. What is less frequently encountered and deserves a more detailed description is how Mie theory can be modified to describe a spherical NP covered by a 2D material. One could, of course, in principle consider a very thin shell, described by an appropriate dielectric function, covering the silicon core [44] and proceed with solving the core-shell geometry. An equivalent alternative, which we describe here, is based on introducing the 2D monolayer by modifying the usual boundary condition for the magnetic field component tangential to the surface of the sphere [40],

$$
\hat{\mathbf{r}} \times\left.\left(\mathbf{H}_{\text {out }}-\mathbf{H}_{\text {in }}\right)\right|_{r=R_{1}}=0,
$$

where $\hat{\mathbf{r}}$ is the unit vector normal to the nanosphere surface and $\mathbf{H}_{\text {in(out) }}$ the magnetic field inside (outside) the sphere. The modification is done by introducing a finite surface current density [80], $\mathbf{J}_{2 \mathrm{D}}$, at the interface ( $\left.r=R_{1}\right)$, such that Eq. (A1) becomes [81]

$$
\hat{\mathbf{r}} \times\left.\left(\mathbf{H}_{\mathrm{out}}-\mathbf{H}_{\mathrm{in}}\right)\right|_{r=R_{1}}=\mathbf{J}_{2 \mathrm{D}} .
$$

The electromagnetic response of the 2D material is thus accounted for via a surface conductivity given by $\sigma_{2 \mathrm{D}}(\omega)=$ $-\mathrm{i} \omega \varepsilon_{0} d\left[\varepsilon_{2 \mathrm{D}}(\omega)-1\right]$, where $\varepsilon_{0}$ is the vacuum permittivity and $d$ is the effective monolayer thickness, usually taken as the layer separation in its parent bulk material; for the specific case of $\mathrm{WS}_{2}$, we take $d=6.18 \AA$ [70]. The surface current density induced by the electric field component tangential to the NP surface, $\left.\mathbf{E}_{\|}\right|_{r=R_{1}}$, can now be obtained from Ohm's law: $\mathbf{J}_{2 \mathrm{D}}=\left.\sigma_{2 \mathrm{D}} \mathbf{E}_{\|}\right|_{r=R_{1}}$.

[1] K. H. Drexhage, J. Luminesc. 1-2, 693 (1970).

[2] Y. Kaluzny, P. Goy, M. Gross, J. M. Raimond, and S. Haroche, Phys. Rev. Lett. 51, 1175 (1983).

[3] G. Rempe, H. Walther, and N. Klein, Phys. Rev. Lett. 58, 353 (1987).

[4] R. J. Thompson, G. Rempe, and H. J. Kimble, Phys. Rev. Lett. 68, 1132 (1992).

[5] J. P. Reithmaier, G. Sęk, A. Löffler, C. Hoffmann, S. Kuhn, S. Reitzenstein, L. V. Keldysh, V. D. Kulakovskii, T. L. Reinecke, and A. Forchel, Nature (London) 432, 197 (2004).

[6] J. Wolters, A. W. Schell, G. Kewes, N. Nüsse, M. Schoengen, H. Döscher, T. Hannappel, B. Löchel, M. Barth, and O. Benson, Appl. Phys. Lett. 97, 141108 (2010).
With the modified boundary condition Eq. (A2), the (electric, $a_{\ell}$, and magnetic, $b_{\ell}$ ) Mie scattering coefficients for a spherical particle coated with an arbitrary 2D material can be obtained [81]:

$$
a_{\ell}=\frac{\varepsilon_{1} j_{\ell}\left(x_{1}\right) \Psi_{\ell}^{\prime}\left(x_{2}\right)-\varepsilon_{2} j_{\ell}\left(x_{2}\right) \Psi_{\ell}^{\prime}\left(x_{1}\right)+f_{\sigma}(\omega) \Psi_{\ell}^{\prime}\left(x_{2}\right) \Psi_{\ell}^{\prime}\left(x_{1}\right)}{\varepsilon_{1} j_{\ell}\left(x_{1}\right) \xi_{\ell}^{\prime}\left(x_{2}\right)-\varepsilon_{2} h_{\ell}^{+}\left(x_{2}\right) \Psi_{\ell}^{\prime}\left(x_{1}\right)+f_{\sigma}(\omega) \xi_{\ell}^{\prime}\left(x_{2}\right) \Psi_{\ell}^{\prime}\left(x_{1}\right)},
$$

$$
b_{l}=\frac{j_{\ell}\left(x_{1}\right) \Psi_{\ell}^{\prime}\left(x_{2}\right)-j_{\ell}\left(x_{2}\right) \Psi_{\ell}^{\prime}\left(x_{1}\right)+f_{\sigma}(\omega) j_{\ell}\left(x_{2}\right) j_{\ell}\left(x_{1}\right)}{j_{\ell}\left(x_{1}\right) \xi_{\ell}^{\prime}\left(x_{2}\right)-h_{\ell}^{+}\left(x_{2}\right) \Psi_{\ell}^{\prime}\left(x_{1}\right)+f_{\sigma}(\omega) h_{\ell}^{+}\left(x_{2}\right) j_{\ell}\left(x_{1}\right)},
$$

where $x_{j}=(\omega / c) \sqrt{\varepsilon_{j}} R_{1}$ (with the subscript $j=\{1,2\}$ denoting the sphere and the host medium, respectively), $c$ is the speed of light in vacuum, and we have assumed that the permeabilities of all media are equal to unity. Moreover, we have introduced the Riccati-Bessel functions $\Psi_{\ell}(x)=$ $x j_{\ell}(x)$ and $\xi_{\ell}(x)=x h_{\ell}^{+}(x)$, where $j_{\ell}$ and $h_{\ell}^{+}$are the spherical Bessel, and Hankel of first kind functions of order $\ell$, respectively. Primes in Eq. (A3) denote derivatives with respect to the corresponding function argument. Lastly, we have defined $f_{\sigma}(\omega)=\mathrm{i} \sigma_{2 \mathrm{D}}(\omega) /\left(\omega \varepsilon_{0} R_{1}\right)$. Setting $f_{\sigma}=0$ returns the standard Mie theory result for a sphere in a homogeneous environment [40].

Both in the standard Mie theory and in its 2D layer variation described here, the extinction and scattering cross sections are calculated through [40]

$$
\begin{aligned}
\sigma_{\text {ext }} & =\frac{2 \pi}{k_{2}^{2}} \sum_{\ell=1}^{+\infty}(2 \ell+1) \operatorname{Re}\left[a_{\ell}+b_{\ell}\right], \\
\sigma_{\text {scat }} & =\frac{2 \pi}{k_{2}^{2}} \sum_{\ell=1}^{+\infty}(2 \ell+1)\left[\left|a_{\ell}\right|^{2}+\left|b_{\ell}\right|^{2}\right],
\end{aligned}
$$

where $k_{2}=(\omega / c) \sqrt{\varepsilon_{2}}$ is the wave number in medium 2. The absorption cross section is straightforwardly obtained from $\sigma_{\mathrm{abs}}=\sigma_{\mathrm{ext}}-\sigma_{\text {scat }}$. Decomposing the cross sections into the corresponding electric (magnetic) dipolar, quadrupolar, etc., contributions means retaining in Eqs. (A4) only the $a_{1}, a_{2}$, etc. $\left(b_{1}, b_{2}\right.$, etc.) terms.

[7] A. Albrecht, A. Retzker, F. Jelezko, and M. B. Plenio, New J. Phys. 15, 083014 (2013)

[8] J. N. Anker, W. P. Hall, O. Lyandres, N. C. Shah, J. Zhao, and R. P. Van Duyne, Nat. Mater. 7, 442 (2008).

[9] W. J. Galush, S. A. Shelby, M. J. Mulvihill, A. Tao, P. Yang, and J. T. Groves, Nano Lett. 9, 2077 (2009).

[10] R. R. Chance, A. Prock, and R. Silbey, Advances in Chemical Physics (Wiley, New York, 1978), Vol. 37, p. 1.

[11] Y. Fu and J. R. Lakowicz, Laser Photon. Rev. 3, 221 (2009).

[12] H. Xu, J. Aizpurua, M. Käll, and P. Apell, Phys. Rev. E 62, 4318 (2000).

[13] A. V. Akimov, A. Mukherjee, C. L. Yu, D. E. Chang, A. S. Zibrov, P. R. Hemmer, H. Park, and M. D. Lukin, Nature (London) 450, 402 (2007) 
[14] D. Martín-Cano, L. Martín-Moreno, F. J. García-Vidal, and E. Moreno, Nano Lett. 10, 3129 (2010).

[15] P. Törmä and W. L. Barnes, Rep. Prog. Phys. 78, 013901 (2015).

[16] M. Nečada, J.-P. Martikainen, and P. Törmä, Int. J. Mod. Phys. B 31, 1740006 (2017).

[17] I. Gerhardt, B. Grotz, P. Siyushev, and J. Wrachtrup, Int. J. Mod. Phys. B 31, 1740004 (2017).

[18] D. G. Baranov, M. Wersäll, J. Cuadra, T. J. Antosiewicz, and T. Shegai, ACS Photonics 5, 24 (2018).

[19] F. Benz, M. K. Schmidt, A. Dreismann, R. Chikkaraddy, Y. Zhang, A. Demetriadou, C. Carnegie, H. Ohadi, B. de Nijs, R. Esteban, J. Aizpurua, and J. J. Baumberg, Science 354, 726 (2016).

[20] J. Bellessa, C. Bonnand, J. C. Plenet, and J. Mugnier, Phys. Rev. Lett. 93, 036404 (2004).

[21] J. Dintinger, S. Klein, F. Bustos, W. L. Barnes, and T. W. Ebbesen, Phys. Rev. B 71, 035424 (2005).

[22] Y. Sugawara, T. A. Kelf, J. J. Baumberg, M. E. Abdelsalam, and P. N. Bartlett, Phys. Rev. Lett. 97, 266808 (2006).

[23] N. T. Fofang, T.-H. Park, O. Neumann, N. A. Mirin, P. Nordlander, and N. J. Halas, Nano Lett. 8, 3481 (2008).

[24] A. González-Tudela, P. A. Huidobro, L. Martín-Moreno, C. Tejedor, and F. J. García-Vidal, Phys. Rev. Lett. 110, 126801 (2013).

[25] G. D. Chatzidakis and V. Yannopapas, J. Mod. Opt. 65, 951 (2018).

[26] J. B. Khurgin, Nat. Nanotechnol. 10, 2 (2015).

[27] G. Kewes, K. Herrmann, R. Rodríguez-Oliveros, A. Kuhlicke, O. Benson, and K. Busch, Phys. Rev. Lett. 118, 237402 (2017).

[28] A. García-Etxarri, R. Gómez-Medina, L. S. Froufe-Pérez, C. López, L. Chantada, F. Scheffold, J. Aizpurua, M. NietoVesperinas, and J. J. Sáenz, Opt. Express 19, 4815 (2011).

[29] A. B. Evlyukhin, S. M. Novikov, U. Zywietz, R. L. Eriksen, C. Reinhardt, S. I. Bozhevolnyi, and B. N. Chichkov, Nano Lett. 12, 3749 (2012).

[30] I. Staude and J. Schilling, Nat. Photonics 11, 274 (2017).

[31] I. Staude, A. E. Miroshnichenko, M. Decker, N. T. Fofang, S. Liu, E. Gonzales, J. Dominguez, T. S. Luk, D. N. Neshev, I. Brener, and Y. Kivshar, ACS Nano 7, 7824 (2013).

[32] D. G. Baranov, D. A. Zuev, S. I. Lepeshov, O. V. Kotov, A. E. Krasnok, A. B. Evlyukhin, and B. N. Chichkov, Optica 4, 814 (2017).

[33] A. I. Kuznetsov, A. E. Miroshnichenko, M. L. Brongersma, Y. S. Kivshar, and B. Luk'yanchuk, Science 354, aag2472 (2016).

[34] S. Jahani and Z. Jacob, Nat. Nanotechnol. 11, 23 (2016).

[35] H. Wang, Y. Ke, N. Xu, R. Zhan, Z. Zheng, J. Wen, J. Yan, P. Liu, J. Chen, J. She, Y. Zhang, F. Liu, H. Chen, and S. Deng, Nano Lett. 16, 6886 (2016).

[36] Q. Ruan, N. Li, H. Yin, X. Cui, J. Wang, and H.-Q. Lin, ACS Photon. 5, 3838 (2018).

[37] S.-D. Liu, J.-L. Fan, W.-J. Wang, J.-D. Chen, and Z.-H. Chen, ACS Photon. 5, 1628 (2018).

[38] P. A. D. Gonçalves, L. P. Bertelsen, S. Xiao, and N. A. Mortensen, Phys. Rev. B 97, 041402(R) (2018).

[39] J. Cuadra, D. G. Baranov, M. Wersäll, R. Verre, T. J. Antosiewicz, and T. Shegai, Nano Lett. 18, 1777 (2018).

[40] C. F. Bohren and D. R. Huffman, Absorption and Scattering of Light by Small Particles (Wiley, New York, 1983).

[41] M. König, K. Busch, and J. Niegemann, Photon. Nanostruct. Fundam. Appl. 8, 303 (2010).
[42] K. Busch, M. König, and J. Niegemann, Laser Photon. Rev. 5, 773 (2011).

[43] R. Chikkaraddy, B. de Nijs, F. Benz, S. J. Barrow, O. A. Scherman, E. Rosta, A. Demetriadou, P. Fox, O. Hess, and J. J. Baumberg, Nature (London) 535, 127 (2016).

[44] S. Lepeshov, M. Wang, A. Krasnok, O. Kotov, T. Zhang, H. Liu, T. Jiang, B. A. Korgel, M. Terrones, Y. Zheng, and A. Alù, ACS App. Mater. Interf. 10, 16690 (2018).

[45] H. Gudjonson, M. A. Kats, K. Liu, Z. Nie, E. Kumacheva, and F. Capasso, Proc. Ntnl. Acad. Sci. 111, E639 (2014).

[46] C. Tserkezis, J. R. Maack, Z. Liu, M. Wubs, and N. A. Mortensen, Sci. Rep. 6, 28441 (2016).

[47] N. Stefanou and A. Modinos, J. Phys.: Condens. Matter 5, 8859 (1993).

[48] K. Busch and C. M. Soukoulis, Phys. Rev. Lett. 75, 3442 (1995).

[49] K. Busch and C. M. Soukoulis, Phys. Rev. B 54, 893 (1996).

[50] S. John, Phys. Rev. Lett. 58, 2486 (1987).

[51] M. Segev, Y. Silderberg, and D. N. Christodoulides, Nat. Photonics 7, 197 (2013).

[52] M. I. Antonoyiannakis and J. B. Pendry, Europhys. Lett. 40, 613 (1997).

[53] M. A. Green, Sol. Energy Mater. Sol. Cells 92, 1305 (2008).

[54] A. Cacciola, O. Di Stefano, R. Stassi, R. Saija, and S. Savasta, ACS Nano 8, 11483 (2014).

[55] T. J. Antosiewicz, S. P. Apell, and T. Shegai, ACS Photonics 1, 454 (2014).

[56] W. Wu, M. Wan, P. Gu, Z. Chen, and Z. Wang, Opt. Express 25, 1495 (2017).

[57] M. J. Gentile and W. L. Barnes, J. Opt. 19, 035003 (2017).

[58] G. Zengin, G. Johansson, P. Johansson, T. J. Antosiewicz, M. Käll, and T. Shegai, Sci. Rep. 3, 3074 (2013).

[59] G. Zengin, T. Gschneidtner, R. Verre, L. Shao, T. J. Antosiewicz, K. Moth-Poulsen, M. Käll, and T. Shegai, J. Phys. Chem. C 120, 20588 (2016).

[60] J. Niegemann, M. König, K. Stannigel, and K. Busch, Photon. Nanostruct. Fundam. Appl. 7, 2 (2009).

[61] $\varepsilon_{\infty}^{\mathrm{Si}}=5.664, f=6.378, \hbar \omega_{1}=3.509 \mathrm{eV}, \hbar \gamma_{1}=0.1702 \mathrm{eV}$; $\omega_{1}$ and $\gamma_{1}$ replace $\omega_{\mathrm{exc}}$ and $\gamma_{\mathrm{exc}}$ in Eq. (1).

[62] P. B. Johnson and R. W. Christy, Phys. Rev. B 6, 4370 (1972).

[63] $\varepsilon_{\infty}^{\mathrm{Au}}=5.819, \quad \hbar \omega_{\mathrm{p}}=8.959 \mathrm{eV}, \quad \hbar \gamma_{\mathrm{p}}=0.052 \mathrm{eV}, \quad f_{\mathrm{L} 1}=$ $0.696, \quad \hbar \omega_{\mathrm{L} 1}=2.765 \mathrm{eV}, \quad \hbar \gamma_{\mathrm{L} 1}=0.622 \mathrm{eV}, \quad f_{\mathrm{L} 2}=$ $1.686, \hbar \omega_{\mathrm{L} 2}=3.438 \mathrm{eV}$, and $\hbar \gamma_{\mathrm{L} 2}=1.183 \mathrm{eV}$.

[64] C. Tserkezis, N. Stefanou, M. Wubs, and N. A. Mortensen, Nanoscale 8, 17532 (2016).

[65] C. Tserkezis, M. Wubs, and N. A. Mortensen, ACS Photonics 5, 133 (2018).

[66] H. Wang, J. Wen, P. Liu, J. Yan, Y. Zhang, F. Liu, J. She, J. Chen, H. Chen, S. Deng, and N. Xu, arXiv:1710.11447.

[67] X. Liu, T. Galfsky, Z. Sun, F. Xia, E.-C. Lin, Y.-H. Lee, S. KénaCohen, and V. M. Menon, Nat. Photonics 9, 30 (2015).

[68] T. Low, A. Chaves, J. D. Caldwell, A. Kumar, N. X. Fang, P. Avouris, T. F. Heinz, F. Guinea, L. Martín-Moreno, and F. Koppens, Nat. Mater. 16, 182 (2017).

[69] G. Wang, A. Chernikov, M. M. Glazov, T. F. Heinz, X. Marie, T. Amand, and B. Urbaszek, Rev. Mod. Phys. 90, 021001 (2018).

[70] Y. Li, A. Chernikov, X. Zhang, A. Rigosi, H. M. Hill, A. M. van der Zande, D. A. Chenet, E.-M. Shih, J. Hone, and T. F. Heinz, Phys. Rev. B 90, 205422 (2014). 
[71] L. C. Flatten, Z. He, D. M. Coles, A. A. P. Trichet, A. W. Powell, R. A. Taylor, J. H. Warner, and J. M. Smith, Sci. Rep. 6, 33134 (2016).

[72] Y. Li, J. D. Cain, E. D. Hanson, A. A. Murthy, S. Hao, F. Shi, Q. Li, C. Wolverton, X. Chen, and V. P. Dravid, Nano Lett. 16, 7696 (2016).

[73] A. Lavie, L. Yadgarov, L. Houben, R. Popovitz-Biro, T.-E. Shaul, A. Nagler, H. Suchowski, and R. Tenne, Nanotechnology 28, 24LT03 (2017).

[74] Y. Li, J. G. DiStefano, A. A. Murthy, J. D. Cain, E. D. Hanson, Q. Li, F. C. Castro, X. Chen, and V. P. Dravid, ACS Nano 11, 10321 (2017).

[75] K. Chen, S.-J. Ding, Z.-J. Luo, G.-M. Pan, J.-H. Wang, J. Liu, L. Zhou, and Q.-Q. Wang, Nanoscale 10, 4130 (2018).

[76] It should be noted that, strictly speaking, the exact position of the optical transition associated with an exciton in a $2 \mathrm{D}$ TMD depends on the dielectric environment surrounding the material. However, since both the band gap and the exciton binding energy decrease with the increase of the environment permittivity (owing to screening), the overall effect in the optical exciton resonance is typically small. Therefore we stress that our analysis should remain valid up to a possible modest shift of the exciton resonance (which in any case may be compensated by adjusting the NP radius to maintain the coupling).

[77] M. Stührenberg, B. Munkhbat, D. G. Baranov, J. Cuadra, A. B. Yankovich, T. J. Antosiewicz, E. Olsson, and T. Shegai, Nano Lett. 18, 5938 (2018).

[78] A. Alù and N. Engheta, Phys. Rev. B 78, 085112 (2008).

[79] C. Tserkezis, N. Papanikolaou, G. Gantzounis, and N. Stefanou, Phys. Rev. B 78, 165114 (2008).

[80] Related to the three-dimensional current density via $\mathbf{J}=$ $\mathbf{J}_{2 \mathrm{D}} \delta\left(r-R_{1}\right)$.

[81] T. Christensen, A.-P. Jauho, M. Wubs, and N. A. Mortensen, Phys. Rev. B 91, 125414 (2015). 